\title{
The Application of D2D Communication Used in Train Control System
}

\author{
Peng Wang ${ }^{1, a}$, Kaicheng $\mathrm{Li}^{2, \mathrm{~b}}$ \\ ${ }^{1}$ National Engineering Research Center of Rail Transportation Operation and Control System, \\ Beijing, 100044, China \\ ${ }^{2}$ National Engineering Research Center of Rail Transportation Operation and Control System, \\ Beijing, 100044, China \\ aemail: 14120290@bjtu.edu.cn, bemail:Kchli@bjtu.edu.cn
}

\begin{abstract}
Keywords: Train to Train Communication; CPN modeling; Train Control System
\end{abstract}
\begin{abstract}
Internet of Vehicles is a research focus of wireless internet transmission, and it is of great significance introducing Internet of Vehicles into railway. This paper introduces the background and significance of the Internet of Train research, and describes the design scheme and key rooting protocols of the system. In this paper, the two trains tracking scenario of the system is chosen modeling and simulating with colored Petri Nets. The quality and function of the new train control system are verified. And also it analysis the effects of Information transmission delay and packet loss rate with different channel quality and different information transmission intervals. At last, this paper provides theoretical basis and suggestions for the real system building.
\end{abstract}

\section{Introduction}

With the development of road transport intelligence and wireless communication technology, device-to-device communication (D2D) comes into being along with Internet of things (IoT) blossoming in the world. The D2D communication in road transport is called VANET, the aim of which is solving the problem of traffic, environment and efficiency, as well as preventing the traffic crashes happening [1]. Comparing with road transportation, railway transportation has higher safety requirements. However, the present train control system is based on train to ground communication and sending movement authority by ground equipment. Thus there will be a lot of hidden danger because of too much ground equipment and complicated interfaces. If bring D2D communication into railway, we can not only reduce the potential risk, but also cut down the system costs, as well as improve the operation efficiency. Therefore, introducing D2D communication into railway is of great significance and development prospect [2]

The research of D2D communication in road traffic field began in the 1980s, and in the later 20 years, Japan and US assigned the special communication frequency for it, which lays the foundation for the research of VANET[4-5]. Now, it is not only the theory research of VANET is rich and deep, but also gradually achieving the commercial, universalness and expand of VANET [6-7]. However the study of train to train (T2T) communication started late that is mostly about physical channel and the MAC layer protocol researching[8-10]. As for practical application of train to train communication, there is no one case except the Alstom's simplified CBTC technology debut in Lille.

This paper introduced a communication protocol design suitable for railway, and described the fault-security policy when communication failure occurs. In the article, it chose a multi-train movement scenario as an example, and a built colored Petri nets model of train to train communication train control system to simulate and analyze the communication performance and system functions. Meanwhile, this paper proposed several suggestions for the establishment of real system. 


\section{The Design of Train Control System Based on Train to Train Communication}

The meaning of train control system based on train to train communication is that though extending the train- ground communication to train-train communication, the trains can independently transmit data without ground equipment. The new system achieves the redistribution of functionality by transforming some ground functions into onboard, thus it can reduce the number of ground equipment and optimize the system structure. Through T2T communication, the train can directly identify the front train's position and determine the movement authority by itself.

Due to the direct communication among trains, the onboard vital computer can directly and continuously get the position and speed of front trains. So that it can reduce the number of ground trackside equipment. This is of great significance for China railway construction for it can not only reduce the security hidden danger, but also cut down the system built cost. However, how to achieve T2T communication is a problem that is urgently to be solved.

According to the characteristics, functions and requirement of T2T communication used in railway field, the structure of T2T train control system is shown in Fig.1. T2T train control system includes mobile nodes (train), database station, relay station, CTC, etc. The main work of train control network is following: realizing the information interaction between train and train, train and CTC, train and CBI, providing safe real-time data for the generation of movement authority, assuring function correctness when train registers, initiates, cancels, normally running, shunts, departures, etc.

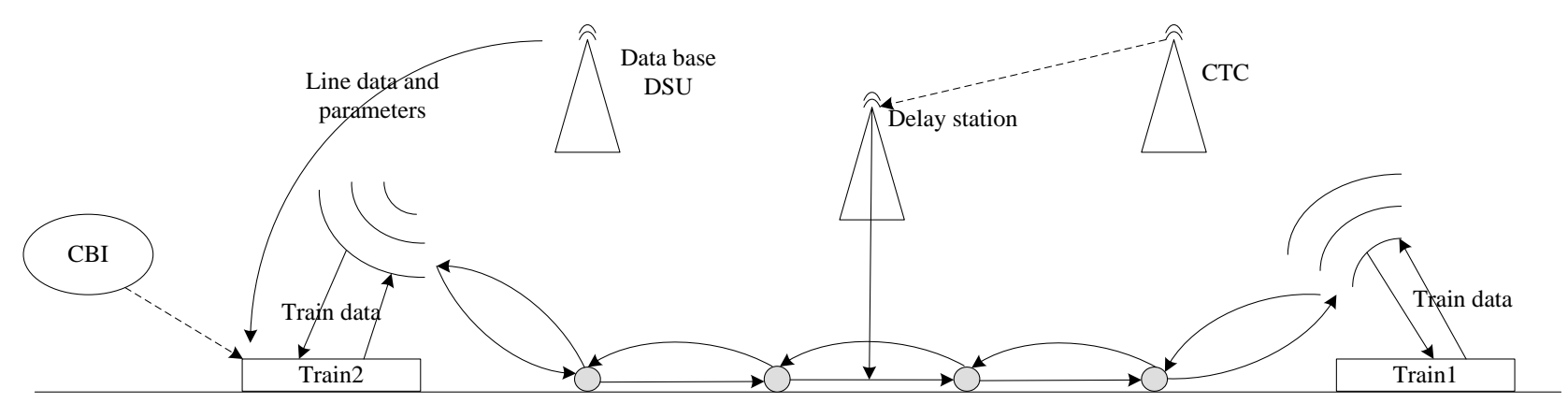

Fig.1. The structure of T2T train control system

Here we choose trains tracking scenario illustrating the work process of T2T train control system. (1) A train requests to establish a connection with the front train. First the information will choose the node that is closest to the behind train by calculating of the unicast routing protocol the unicast routing protocol (GPSR). Then the information will be transmitted through other fixed nodes according to the direction of the train moving. The mode of transmission is Multi-hop. (2) When the front train has gotten the request from the train behind, it sends agreement to the back train, the information transmitting process is the same as step 1 except the direction of information transmission is opposite. (3)After the train behind receive the agreement from the front train, it confirms the message and ask for the position of the front train. (4) The front train send its real-time position, which means entering the data transmission normally. If there is a communication failure, according to the fault - security principle, the work process is: (1) the train takes full service braking until the train stops. (2)The train gets connection with CTC after the train stops for two minutes. (3) The train asks for OS driving. (4) The train check the communication condition every moment when it runs in OS mode. The sequence diagram of T2T communication is shown in Fig.2. 


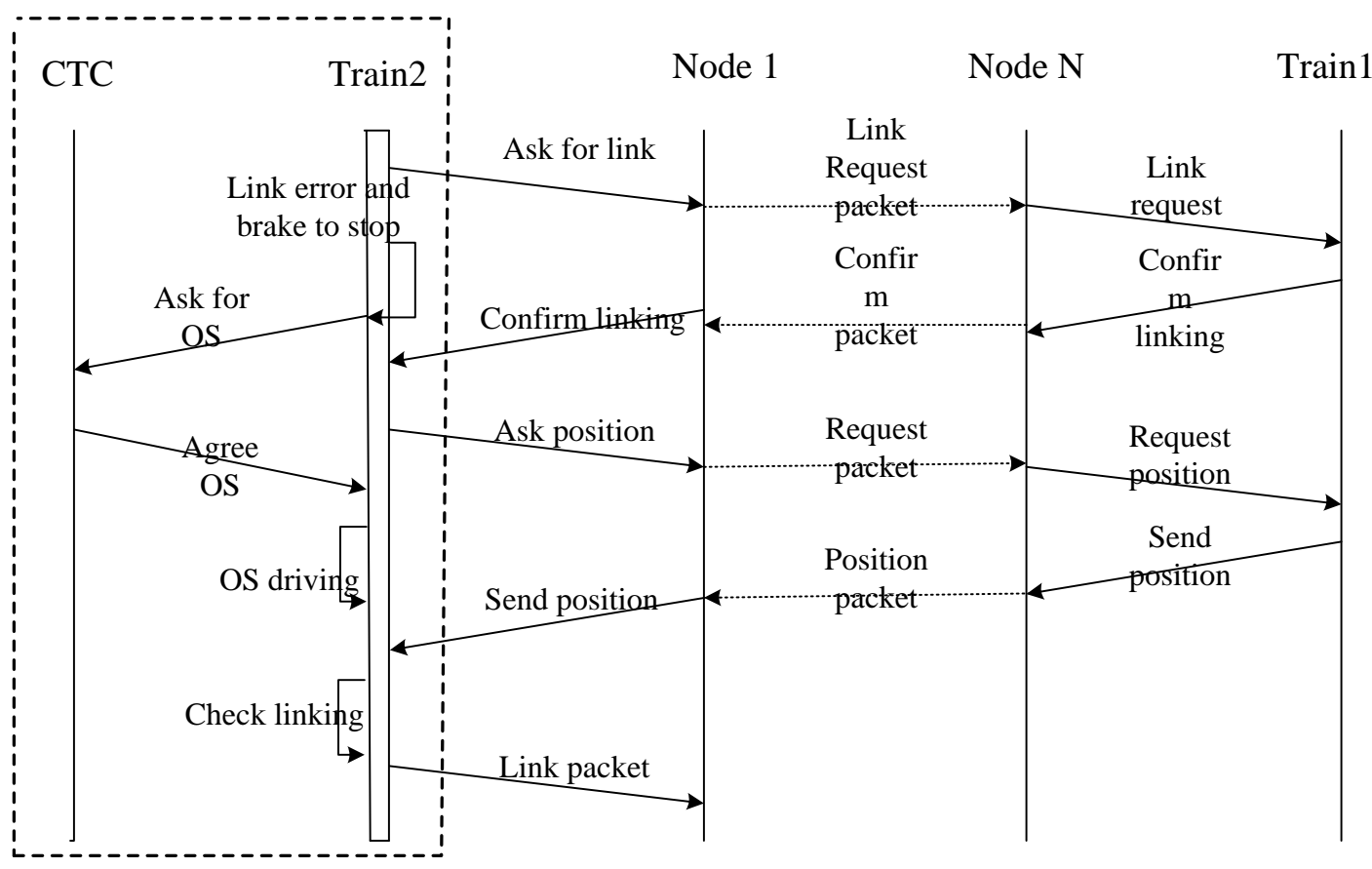

Failure

Normal

Fig.2. The sequence diagram of $\mathrm{T} 2 \mathrm{~T}$ communication process

\section{The Modeling of T2T Train Control System}

In this paper, we chose the scenario trains tracking to build the CPN model according to the design scheme of T2T train control system. In this scenario, the modeling of whole communicate process adopted parting-module way to do the research and the whole model could be divided into three parts: sending module modeling, codes transmitting module modeling and message receiving module modeling.

When the train get connected with the front train, the message from the front train need to be send in course. The steps are listed below. (1) The train data should be copied to the packet sending module in order to finish the synchronization of complete information. (2) This module need to confirm the sending context by checking the timestamp in real system which is replaced by variable ' $n$ ' in this modeling and simulation. Every circulation of sending and receiving, $n$ will be plus one and waiting for next round. (3) The front train sends message and the train behind receives the message. (4)This module need keep record about the sending and receiving.

After the message is sent, the fixed nodes will receive, judge and retransmit it. The steps of this part are following: (1) The fixed node receive and decode the packet and the front train information. (2) The fixed node compares ' $n$ ' which is on behalf of timestamp with preset value ' $k$ ' in order to check whether the information is effective. (3) According to the checking results, change the value of ' $n$ ' and ' $k$ ' and send the feedback for the next round sending. (4) If the receiving message is right, the fixed code store and forward the message.

Then the message packet is transmitted from the fixed code, the message receiving module of the second train will receive and save the message, this process is similar with fixed codes receiving packets. And the whole CPN model is shown in Fig.3. 


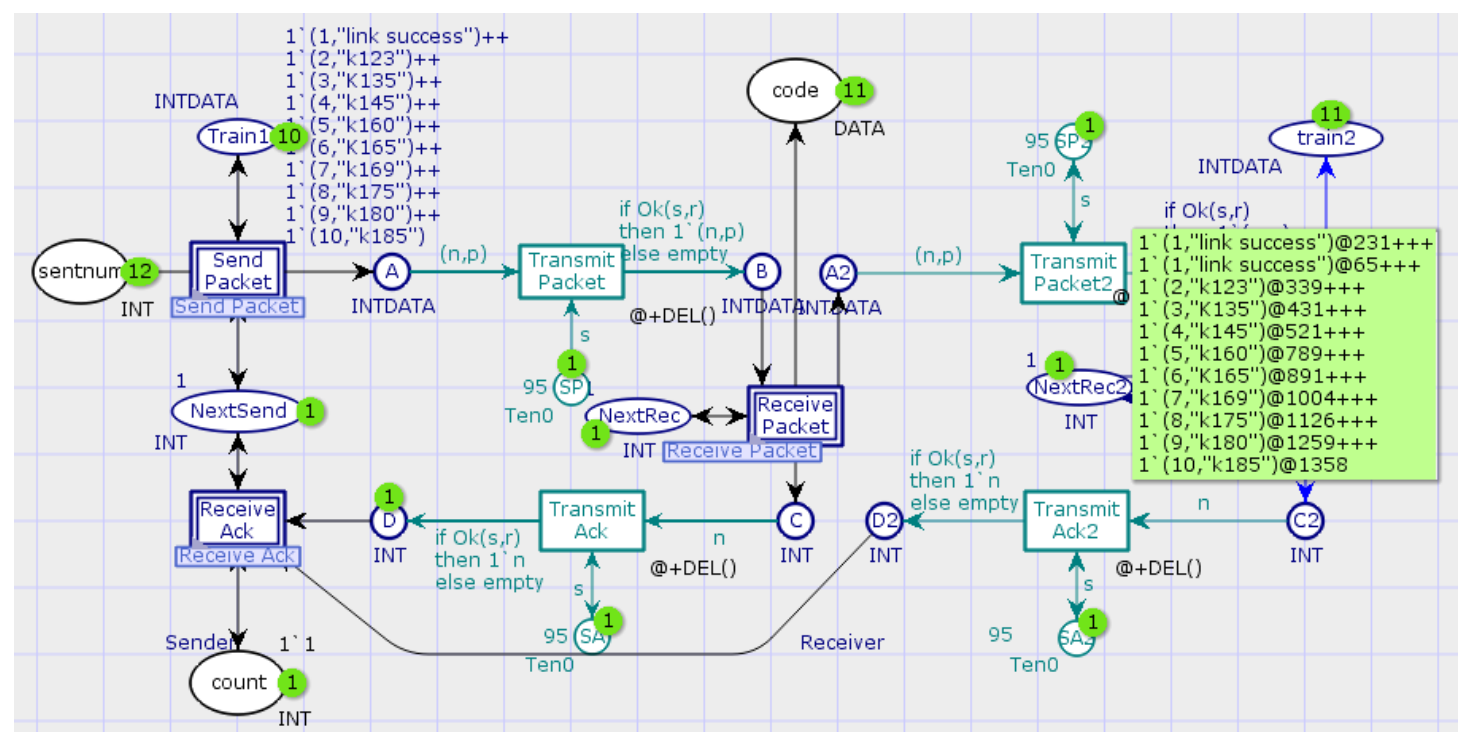

Fig.3. T2T communicate simulation

\section{Test results}

In this simulation, it can set different failures to make train2 couldn't receive the information from train1. We could observe the system working process and behavior in different condition. The simulation results are following: (1) The flag bit is 1 if one of 'link1' and 'link2' is normal and train2 could get all messages. (2) If none of 'link1' and 'link2' is normal, train 2 couldn't get train1 information, but it can stop on time and turn to OS when it stops two minutes later. And CTC or other trains can get train1 position, lest affect other trains' operation. So this model can protect the train from crash and this T2T basic scheme is safe enough to ensure the train running.

Figure 4 shows the delivery rates in different conditions and the rates come from 100 times simulation each channel quality. From the results we can see the delivery rate will lift with the channel quality rise. The trends tend to be linear.

The design suggestions of actual system: It is necessary to choose optical fiber astransmission media among fixed codes for there is nearly no loss using optical fiber. But the channel quality between trains is not easy to control, therefore it reinforces that effective retransmission algorithm is needed, so that the message can be received though the loss rate is high.
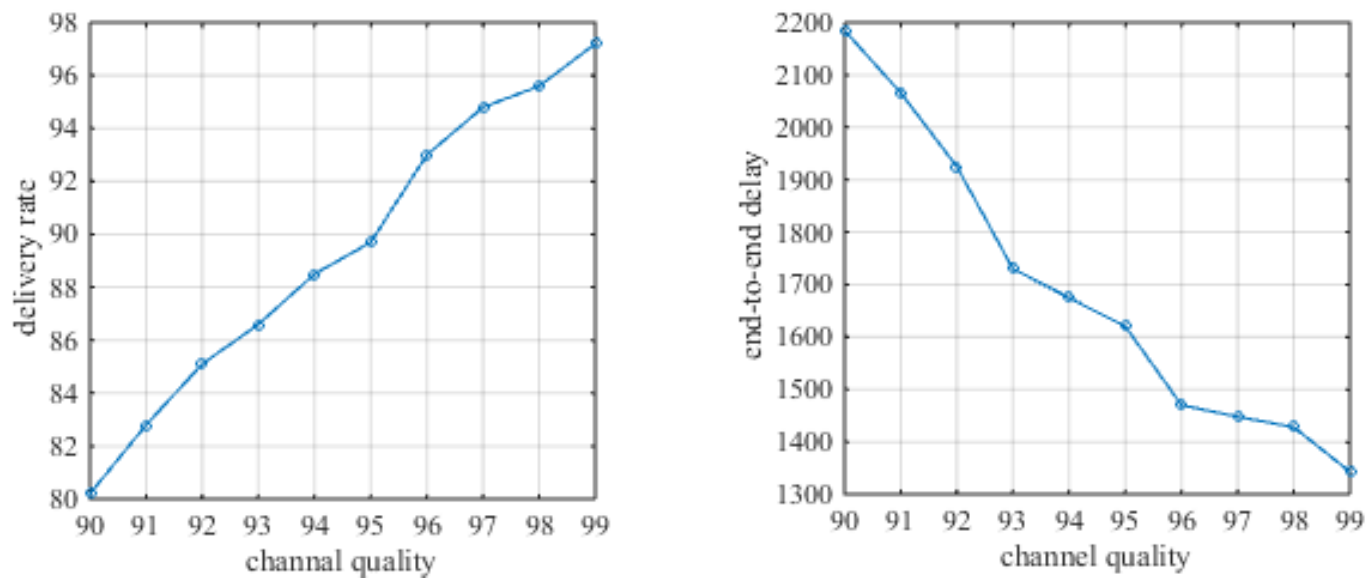

Fig.4 The delivery rate and end-to-end delay in different channel quality

Figure 4 also shows that the delay is strongly influenced by the channel quality. $10 \%$ of the channel quality change causes nearly double end-to-end delay. The design suggestions of actual system: although the times of retransmission determines the delay time, but retransmission intervals also impact the whole messages transferring time, which is to say infinite fast retransmission is certain to reduce the delay time. However, the interval of retransmission can't be infinitesimal. The interval of retransmission is shown in table 1 . Synthesizes relay and network overhead, $150 \mathrm{~ms}$ is the 
best interval of retransmission.

Table 1 the effect of different sending interval to delay

\begin{tabular}{l|l|l|l|l|l}
\hline Interval time/ms & $50 \mathrm{~ms}$ & $100 \mathrm{~ms}$ & $150 \mathrm{~ms}$ & $200 \mathrm{~ms}$ & $250 \mathrm{~ms}$ \\
\hline Average of delay/ms & 1195.7 & 1307.7 & 1499.6 & 1623.8 & 1964 \\
\hline Average number of packets & 30.4 & 20.5 & 13.2 & 11.9 & 11.1 \\
\hline
\end{tabular}

\section{Conclusion}

Based on the analysis of the mechanical theory as the foundation, designed the soccer robot pick the ball institutions optimal design process, found aim function, select design variables and the corresponding optimization algorithm to optimize a complete set of institutions. At last through the test to get the final performance parameters of the institution. Experiments show that the system has higher accuracy and stability, the new optimize pick the ball have design basic requirements, and achieved good ideal control effect.

\section{Acknowledgement}

In this paper, the research was sponsored by the Beijing Jiaotong University basic research costs (Project No. 2015JBM014).

\section{References}

[1] Su Jing, Wang Dong, Zhang Fei-fei. Review of vehicle networking technology application [J]. Intelligent Processing and Application, 2014, 06: 69-12.

[2] Hartenstein H, Bochow B, Ebner A, et al. Position-aware ad hoc wireless networks for inter-vehicle communications: the Fleetnet project[C]//Proceedings of the 2nd ACM international symposium on Mobile ad hoc networking \& computing. ACM, 2001: 259-262.

[3]Cheng Gang, Guo Da. The research of VENAT developing and current situation [J]. Mobile Communication, 2011, 35(17):23-26.

[4] Wang Zhe. A study on performance analysis of wireless inter- vehicle communication system in traffic streams [D]. Hefei: USTC, 2009.

[5] Liu Xue. The propagation model study based on intelligent traffic safety vehicle-to-vehicle wireless communication system [D]. Beijing: Beijing Jiao Tong University, 2010.

[6] Wang Zhao-ran, Xie Xian-zhong, Zhao Ding-xin. Key technique of vehicular Ad Hoc Network [J]. Science Technology. 2011, 27(1): 44-51.

[7] Yang Qiong, Shen Lian-feng. System architecture and communication protocols in vehicular Ad Hoc networks [J]. ZTE Communications. 2011, 03:8-11.

[8] García C R, Lehner A, Strang T, et al. Channel model for train to train communication using the $400 \mathrm{MHz}$ band[C]//Vehicular Technology Conference, 2008. VTC Spring 2008. IEEE. IEEE, 2008: 3082-3086.

[9] Liu P, Ai B, Zhong Z, et al. A Novel Train-to-Train Communication Model Design Based on Multihop in High-Speed Railway[J]. International Journal of Antennas and Propagation, 2012, 2012.

[10] Chen Qi-xiang, Li Mao-qing, Lin Jun-ting. Research on train-to-train direct communication technology based on Ultrashort Wave [J]. Computer Engineering. 2013 (12):5-10. DOI:doi:10.3969/j.issn.1000-3428.2013.12.002. 